\title{
The Applicability Analysis of IPv6 Translation Transition Mechanisms
}

\author{
Wei Mi, Xiaodan Zhang \\ Computer Network Information Center, Chinese Academy of Sciences, Beijing, China \\ Email: miwei@cstnet.cn, zhangxiaodan@cstnet.cn
}

Received March 2015

\begin{abstract}
Due to the exhaustion of IPv4 address resources, the transition from IPv4 to IPv6 is inevitable and fairly urgent. Numerous transition mechanisms have been proposed to solve challenging issues of IPv6 transition. An inter-connection between IPv4 and IPv6 networks or hosts requirement has been happening throughout the IPv6 transition process. And one-time translation scheme is indispensable to achieve the inter-connection. In addition, double translation can be used in the IPv4-IPv6-IPv4 scenario. As a long-term strategy, translation scheme is important and inevitable. However, because of the diverse characteristics and transition requirements of practical networks and the lack of applicability analysis, the selection and deployment of transition mechanisms are facing with grand challenges. Targeting at those challenges, this paper investigates the basic issues and key elements of IPv6 translation transition mechanisms, and presents its first applicability index system. In particular, we analyze the applicability of existing proposed translation techniques based on the presented index system, which has significant guidance in the practical deployment of IPv6 transition techniques.
\end{abstract}

\section{Keywords}

IPv6 Transition, Translation Mechanisms, Applicability, Index System

\section{Introduction}

With the rapid growth of Internet scale, the exhaustion of IPv4 addresses is a significant problem. IPv6 was designed to be an evolutionary step from IPv4, overcoming the problems of IPv4 and promoting the development of Next Generation Internet. Due to the incompatibility in nature, IPv6 transition will face many technical challenges, such as heterogeneous addressing, different semantic, routing isolation, huge size and the transparent to users and to applications. IPv6 transition is a world recognized significant technology problem in the development of Next Generation Internet.

During the process of IPv6 transition, no matter which network protocol is used, it must support both IPv4 services and IPv6 services, and ensure the transparence to the upper layer applications. The characteristics and transition requirements of practical networks are diverse and complex. Theoretically, the IPv6 transition scenarios can be decomposed into two types: inter-connection (IPv4-IPv6) and heterogeneous traversing (IPv6-IPv4IPv6 or IPv4-IPv6-IPv4). An inter-connection happens when networks or hosts using different address families 
are directly connected and want to communicate. A traversing happens when two or more native IPv4/IPv6 networks (or hosts) are separated by a network which uses the other address family and thereby is not IPv4/ IPv6-capable.

Numerous transition mechanisms have been proposed to solve challenging issues of IPv6 transition, which can be divided into dual-stack, tunnel, and translation mechanisms. Dual-stack mechanisms can support both IPv4 and IPv6, but they bring the high cost on both the hardware upgrading and network operation/management. Tunnel mechanisms possess the merits of expansibility, flexibility and simplified achievement. And the Softwire Working Group of IETF is focusing on developing and standardizing the tunnel mechanisms. However, tunnel scheme cannot resolve the inter-connection problem which has been happening throughout the IPv6 transition process. Compared with dual-stack and tunnel, translation can be used to achieve direct communication between IPv4 and IPv6. After development these years, translation technology has already formed its own one more complete system which had been developed and standardized in the Behave Working Group of IETF. As a long-term strategy, translation scheme is important and inevitable.

In practical deployment, it is important to find feasible transition mechanisms and make appropriate plan to cover all potential communication scenarios. However, it brings great challenges to the research community of IPv6 transition. With the diverse characteristics and transition requirements of practical networks and the lack of overall transition architecture, the selection and deployment of IPv6 transition mechanisms are very difficult. Thus, there is a strong need to take the research on the applicability of transition mechanisms. However, the applicability criterion and analysis are lack in the current literature review.

In an effort to push forward the IPv6 transition process, this paper deeply discusses the basic issues and key elements of IPv6 translation mechanisms, and presents the first applicability index system and analyzes the applicability of existing translation techniques. All of these have guiding significantly in the IPv6 transition process.

The rest of this paper is organized as follows. Section 2 presents the related work. The applicability index system and the applicability analysis of mainstream IPv6 translation mechanisms are shown in Section 3 and 4. Finally, Section 5 concludes this study.

\section{Related Work}

Numerous studies on the evaluation of IPv6 transition mechanisms have been reported in the current literature. Shin et al. [1] showed the impact of IPv6 transition mechanisms on user applications. Law et al. in [2] focused on the performance of dual-stack technologies in terms of various network metrics including network connectivity, hop-count, RTT, throughput, operating systems dependencies and the address configuration latency. The authors in [3] [4] provided the evaluation of tunnel mechanisms with the key performance-related metrics including throughput, delay, jitter, and the CPU usage of transition nodes. AlJa'afreh et al. [5] gave the comparison between the tunneling process and mapping schemes for IPv4/IPv6 transition using end-to-end delay and throughput as the key performance metrics. Guerin and Hosanagar [6] adopted a simple model to illustrate how the connectivity quality affects both IPv6 adoption and the volume of translation traffic, and summarize their implications for IPv6 adoption. The authors in [7] evaluated the dual-stack protocol and tunneling transition based on the metrics of throughput and round-trip delay. Several studies [8]-[11] presented the comparisons of translation mechanisms with the aspects of operation complexity and scalability, real-time communications, field device, multicast address, and application-layer protocol. The authors in [8] proposed the evaluation of the transition mechanisms including the estimation on the scalability, heterogeneous addressing and applicationlayer translation, hardware cost, performance and capacity of the equipment, security, end-to-end property, and the influence for developing applications.

Compared with existing studies, in [12], we also provided unified assessment criterion in terms of functionality, applications, performance, development and security to evaluate the mainstream transition mechanisms. The unified evaluation criterion is shown in Table 1.

\section{The Applicability Index System}

\subsection{Basic Problems}

Translation scheme is used to achieve direct communication between IPv4 and IPv6 networks (or hosts). Its basic operation is to convert the semantics between IPv4 and IPv6, turning IPv4 packet into IPv6 if the packet is 
Table 1. The evaluation criterion of IPv6 transition mechanisms.

\begin{tabular}{cc}
\hline Evaluation criterion & Description \\
\hline $\begin{array}{l}\text { Functionality } \\
\text { Applications }\end{array}$ & $\begin{array}{r}\text { Including the transition scenario, transition function, equipment requirements, } \\
\text { and IPv4 and IPv6 address requirements. } \\
\text { The impact on IPv4 or IPv6 application. }\end{array}$ \\
$\begin{array}{l}\text { Performance } \\
\text { Teployment } \\
\text { Security }\end{array}$ & $\begin{array}{r}\text { The performance evaluation is mainly for the equipments, such as forwarding performance, } \\
\text { and the searching, storage, and computational overhead. }\end{array}$ \\
\hline
\end{tabular}

destined to IPv6 network, or turning IPv6 packet into IPv4 if the packet is destined to IPv4 network.

- The basic data operation

IPv4-IPv6 packet translation is the basic data plane operation. It involves network, transport, and application layer. Thus, the basic operations include address and port conversion, IP/TCP/UDP protocol field translation, and application layer translation (address and port conversion when they appear in application protocol). What is more, to overcome further diversities in the protocol definition between IPv4 and IPv6, translation has to take care of issues like fragmentation and reassembling, path MTU (Maximum Transmission Unit) discovery, ICMP, etc.

- The basic control operation

The basic control plane operation is the address conversion rule: either some special address scheme needs to be deployed in advance, or dynamic address bindings have to be built during the translation. Heterogeneous addressing (learning the in-protocol address of the remote end) and corresponding routing should be performed based on the address conversion rule.

- The translation model

According to the applicable scenarios, the models of IPv6 translation mechanisms are divided into application-side, network-side and host-side translation. According to the address conversion manner, we can also divide network-side translation mechanisms into stateless translation and stateful translation.

\subsection{Key Elements}

- Transition equipment

In translation technologies, the translator is the transition equipments. Usually, network-side translation happens on the IPv4-IPv6 border, so the translator would be an AFBR (Address Family Border Router). And host-side translation happens in the TCP/IP stack of the end host, so the translator would be host. They should support address and port conversion, IP/TCP/UDP protocol field translation and also maintain the state. Thus, translator has requirements in the use of bandwidth, computing and finding, storage.

- Address translation

Using specific address conversion rules, the translator either gets IPv4 address from a specific position of IPv6 address, or builds the IPv6 address using IPv4 address. The address conversion rule includes some special address scheme needs to be deployed in advance, and dynamic address bindings have to be built during the translation.

- Other fields translation

Except the source address and destination address, there are other fields (fragmentation ID, checksum, banfragmentation flag, etc.) in IP/ICMP packets, which carry specific information. In the IPv4-IPv6 translation process, the header information of IPv4 packet cannot be lost. Therefore, translation techniques are required to ensure the integrity of IPv4 information.

- State maintenance

In IPv6 transition, the state is available to only represent a series of attributes mapping relationship among a communication entity (such as client, server), a communication path (the connection between communication entities), or a communication process (control flow and data flow). The IP address in network layer and ID in transport layer of the communication entities are the core state which need translation techniques to maintain. 


\section{- DNS64 and DNS46}

The main function of DNS64 and DNS46 is to realize the bi-directional translation between A and AAAA record. DNS64 translates the AAAA query from IPv6 hosts into A query when receiving one, and DNS46 translates the A response for IPv6 hosts into AAAA response following the IPv4-mapped address rule before sending one out. There are usually two kinds of implementations: static configuration DNS records and dynamic translation.

- Application layer translation

Those applications whose address and port conversion when they appear in application protocol cannot work in NAT and IPv4/IPv6 translation environments. Such as FTP, SIP, etc. One solution is assist applications to realize translation work by using the application layer gateway.

\subsection{Applicability Index System}

Based on the analysis of basic problems and key elements, we built the first applicability index system in terms of sustainable, applications, performance and development to evaluate all IPv6 translation transition mechanisms. The applicability index system is shown in Table 2.

\section{The Applicability Analysis}

In the all stages of transition from IPv4 to IPv6, IPv4 networks/hosts and IPv6 networks/hosts are likely to coexist. For the operators, supporting inter-connection is inevitable. One-time translation mechanisms emerged as required.

Table 2. The applicability index system.

\begin{tabular}{|c|c|c|c|}
\hline \multicolumn{3}{|c|}{ Applicability criterion } & Description \\
\hline \multirow{2}{*}{ Sustainable } & \multicolumn{2}{|c|}{ Scenarios and function of transition } & $\begin{array}{l}\text { Whether meet the needs of } \\
\text { transitional scenario. }\end{array}$ \\
\hline & \multicolumn{2}{|c|}{$\begin{array}{l}\text { The coupling degree between IPv4 address and IPv6 address. } \\
\text { The reuse rate of IPv4 addresses resource. }\end{array}$} & $\begin{array}{l}\text { Whether promote the deployment } \\
\text { and usage of IPv6. }\end{array}$ \\
\hline \multirow{2}{*}{$\begin{array}{l}\text { The support degree } \\
\text { of business application. }\end{array}$} & \multicolumn{2}{|c|}{ The support degree of IPv4 application. End-to-end property. } & $\begin{array}{l}\text { Impact on the IPv4 business } \\
\text { application. }\end{array}$ \\
\hline & \multicolumn{2}{|c|}{ The support degree of IPv6 application. } & $\begin{array}{l}\text { Impact on the IPv6 business } \\
\text { application. }\end{array}$ \\
\hline \multirow{6}{*}{ Performance } & $\begin{array}{l}\text { The performance requirement of } \\
\text { translator }\end{array}$ & $\begin{array}{c}\text { The routing information } \\
\text { announcement } \\
\text { The space and time overhead } \\
\text { of state maintenance }\end{array}$ & $\begin{array}{l}\text { The capacity of bandwidth, } \\
\text { computing and finding, storage. }\end{array}$ \\
\hline & Routing scalability & $\begin{array}{l}\text { The aggregation of } \\
\text { IPv6 addresses }\end{array}$ & Impact on the scope of deployment. \\
\hline & \multicolumn{2}{|c|}{ Robustness } & The capacity of redundancy backup. \\
\hline & Technological and industry maturity & The support degree of IETF & The support degree of standard \\
\hline & & Impact on application layer. & \\
\hline & Update cost & Impact on network layer. & The impact on the present network. \\
\hline \multirow[t]{4}{*}{ The cost of development } & & Impact on end users layer. & \\
\hline & & Configuration & \\
\hline & $\begin{array}{l}\text { The cost of operation, management } \\
\text { and maintenance }\end{array}$ & Maintenance & It impact on operator. \\
\hline & & Troubleshooting & \\
\hline Security & \multicolumn{2}{|c|}{ Including the security issues and concerns. } & $\begin{array}{l}\text { The security of translation } \\
\text { mechanisms. }\end{array}$ \\
\hline
\end{tabular}


With the IPv6 development, IPv4 Internet has been gradually replaced. For the low cost, network operators tend to build IPv6 network rather than dual-stack network. In order to ensure the compatibility of legacy IPv4 application, IPv4-IPv6-IPv4 double translation scheme is provided.

According to the different transition stages, this paper will analyze the applicability of one-time translation mechanisms in the all stages and double translation mechanisms in the middle- to-late-stage.

\subsection{The Applicability Analysis of One-Time Translation Mechanisms}

IETF has developed and standardized many one-time translation transition mechanisms, such as network-side translation mechanisms which can be divided into stateless translation (SIIT [13], IVI [14]) and stateful translation (NAT-PT [15], NAT64 [16]), and host-side translation mechanisms (BIH [17]). In this section, we will analyze the applicability of these mechanisms, which is summarized in Table 3.

SIIT is an early stateless translation mechanism. It does not introduce new security issues to the network. However, the usage of fixed address prefix brings significant routing scalability problem. Due to the per-host IPv4 address consumption requirement, the IPv6 side of SIIT cannot be huge which is unfavorable to IPv6 transition. Therefore, SIIT apply to the early-stage of IPv6 transition.

IVI follows the principle of stateless translation and improves SIIT. The use of NSP (network-specific, variable prefix) makes the routing scalability is no longer a concern. However, the per-host IPv4 address consumption is still required in IVI. Therefore, the IPv6 side cannot be huge and IVI also apply to the early-stage of IPv6 transition.

NAT-PT is originally adopted to achieve the heterogeneous addressing procedure for both sides with DNSALG on the translator. However, it possesses several technical issues, e.g., the disruption of end-to-end transparency, information loss and misunderstanding of DNS-ALG, low scalability of network topology caused by the deep-coupling between DNS-ALG and translator, and the inability to redirect traffic with persistence addressmapping state.

As an enhanced mechanism, NAT64 extracts the DNS-ALG function from the translator and makes it become a dedicated DNS64 server. However, NAT64 only specifies the communication initiated from the IPv6 side. It also destroys the end-to-end transparency. NAT-PT and NAT64 have DoS attack on the binding table, with ingress filtering on the IPv6 side as the solution.

In the host-side translation mechanisms, the translation is inside the TCP/IP stack of the host, which may reduce the deployment overhead of network. However, due to the scale of terminal hosts is much larger than the device in the network side, it will bring a great pressure to ISP and be unfavorable to IPv6 transition.

\subsection{The Applicability Analysis of Double Translation Mechanisms}

At present, IETF has been developing and standardizing double translation transition mechanisms. The mainstream mechanisms include 464XLAT [18], dIVI [19] and MAP-T [20]. The applicability analysis of these mechanisms is provided and summarized in Table 4.

464XLAT supports an IPv4 host (especially mobile host) to connect to the IPv4 Internet through the IPv6 network. Due to the combination of SIIT on the user side and NAT64 on the carrier side, 464XLAT faces the issues in both SIIT and NAT64.

As an extension of IVI, dIVI is a stateless network-side translation in which one IPv4 address is shared by multiple IPv6 hosts through port space division. However, dIVI cannot realize the inter-connection between two IPv4 hosts insider the two dIVI network separately.

In MAP-T, the IPv6 packets have to use the IPv4-mapped addresses as source addresses in the inbound direction and destination addresses in the outbound direction. However, since the mechanism deeply couples IPv4 and IPv6 addressing, it becomes a little less flexible to deploy: the deployment has to be entire-network style rather than on demand style, otherwise some of the coupled IPv4 addresses will be wasted.

\section{Conclusion}

For the consideration of deployment scenarios and address format, numerous translation transition mechanisms have been proposed in IETF. However, due to a wide range of mechanisms and a lot of overlap and similar 
Table 3. The applicability analysis of one-time translation mechanisms.

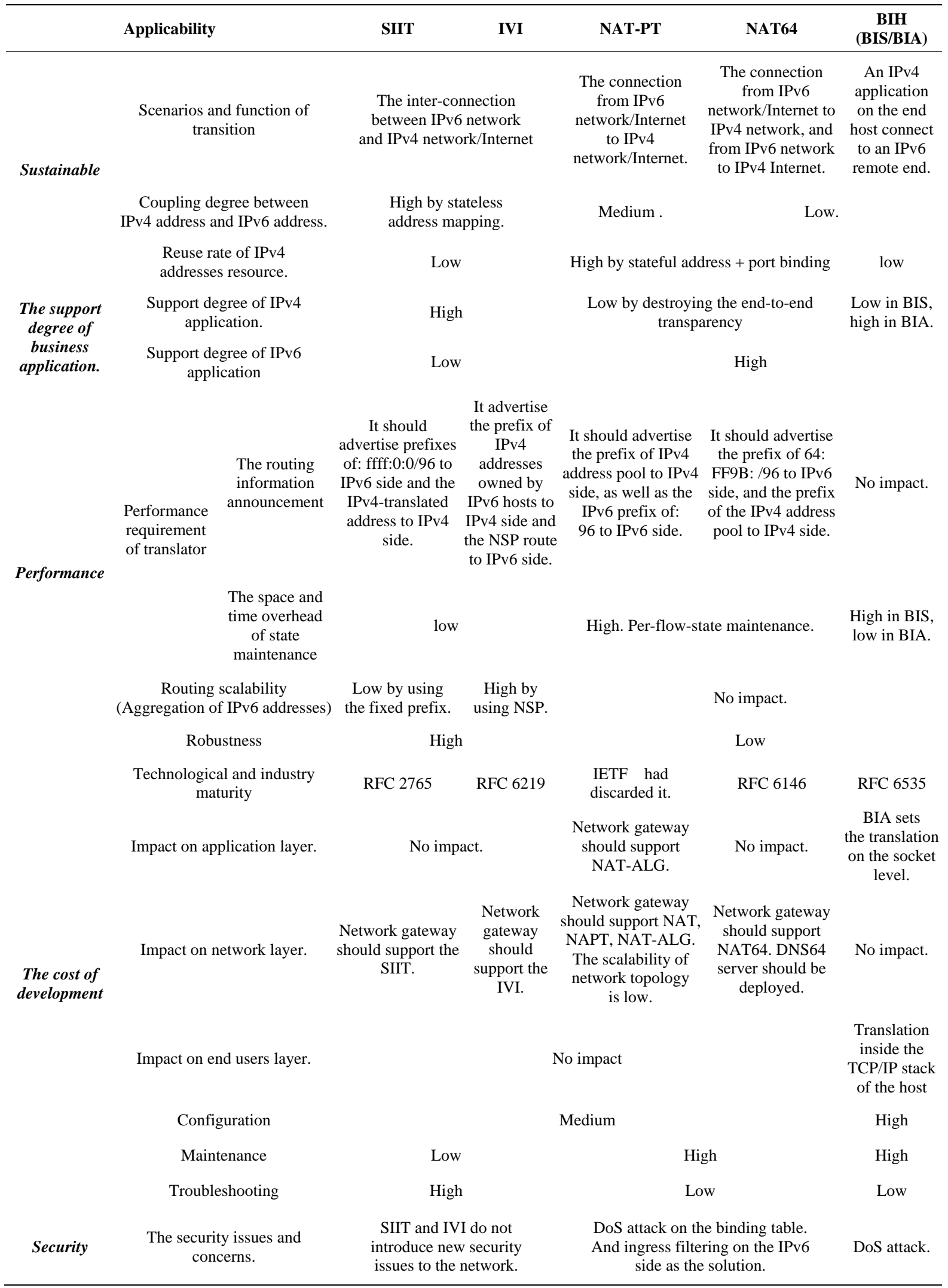


Table 4. The applicability analysis of double translation mechanisms.

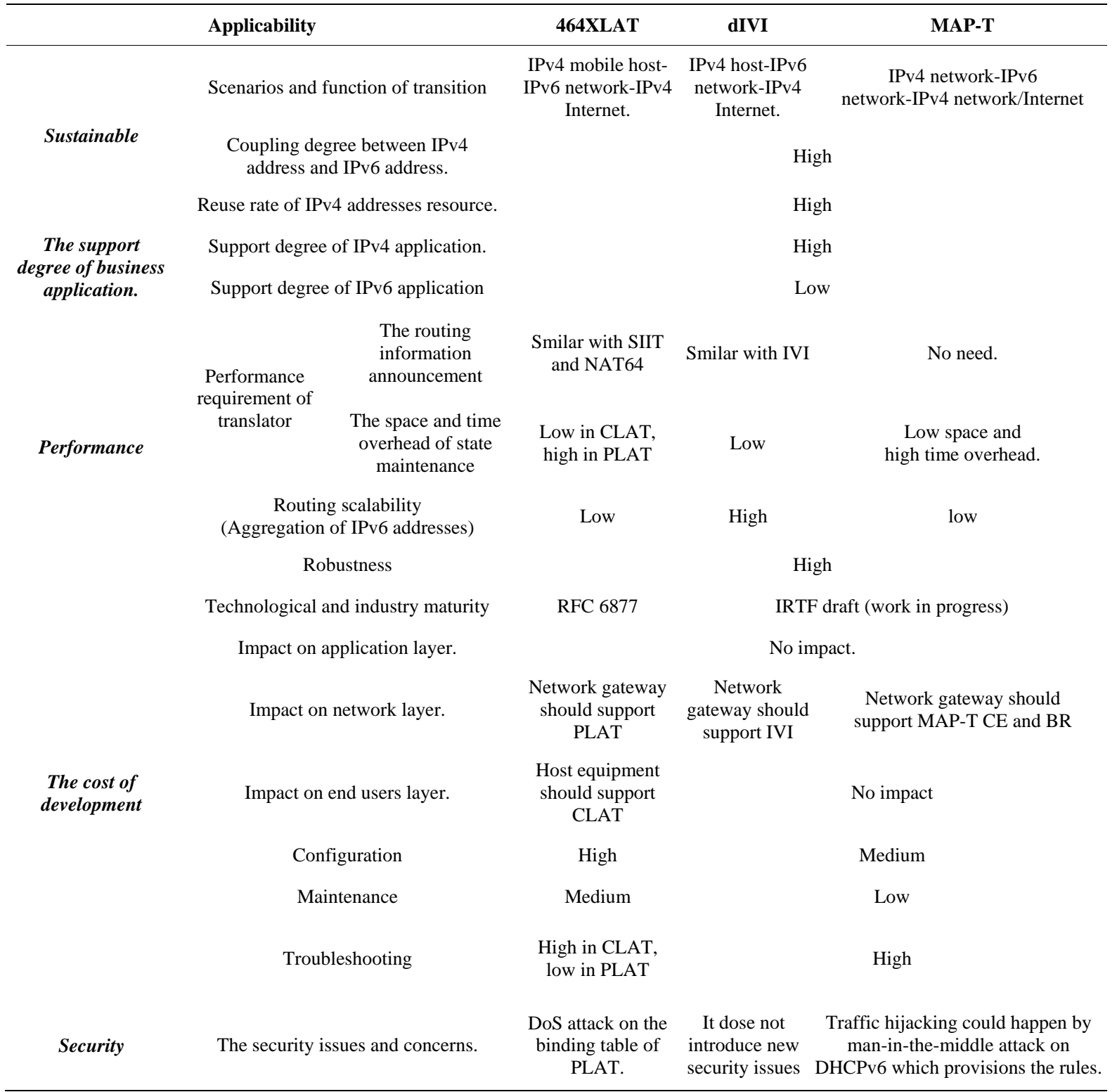

functions, no one translation mechanism can be used in all transition scenarios. This paper has provided the first applicability index system, and highlighted the applicability of all IPv6 translation mechanisms to help the operators decide on the development scheme for their IPv6 transition.

We can observe from the applicability analysis that 1) network-side translation leads less overhead to ISP than host-side translation, and the mainstream of translation techniques is network-side translation; 2) stateless network-side translation requires voracious consumption of IPv4 addresses, while stateful translation requires per-flow state maintenance, and both are not suitable for large scale networks; 3) Among the network-side translation mechanisms, IVI is a feasible stateless translation mechanism, and NAT64 is a feasible stateful translation mechanism; 4) 464XLAT is able to resolve the heterogeneous traversing problem of IPv4 mobile host to IPv4 Internet, and MAP-T is more applicable to the heterogeneous traversing between IPv4 networks.

\section{Acknowledgements}

We are grateful for Jingguo Ge from the Computer Network Information Center of Chinese Academy of 
Sciences, who gave us kind encouragement and useful instructions all through our writing.

Furthermore, this work is supported by the National High-tech R\&D Program of China (863 Program) under Grant No. 2011AA01A101; the National Key Technology Research and Development Program of the Ministry of Science and Technology of China under Grant No.2012BAH01B00; the National Program on Key Basic Research Project of China (973 Program) under Grant No. 2012CB315800; the Strategic Priority Research Program of the Chinese Academy of Sciences under grant No. XDA06010201.

\section{References}

[1] AlJa'afreh, R., Mellor, J. and Awan, I. (2009) A Comparison between the Tunneling Process and Mapping Schemes for IPv4/IPv6 Transition. International Conference on WAINA’09, IEEE Press, Bradford, 601-606. http://dx.doi.org/10.1109/WAINA.2009.209

[2] Law, Y.N., Lai, M.C., Tan, W.L. and Lau, W.C. (2008) Empirical Performance of IPv6 vs. IPv4 under a Dual-Stack Environment. IEEE International Conference on ICC’08, IEEE Press, Beijing, 5924-5929.

[3] Aazam, M., Syed, A.M., Khan, I. and Alam, M. (2011) Evaluation of 6to4 and ISATAP on a Test LAN. IEEE Symposium on ISCI, IEEE Press, Kuala Lumpur, 46-50. http://dx.doi.org/10.1109/ISCI.2011.5958881

[4] Gilligan, R. and Nordmark, E. (1996) Transition Mechanisms for IPv6 Hosts and Routers. IETF RFC 1933.

[5] Guerin, R. and Hosanagar, K. (2010) Fostering IPv6 Migration through Network Quality Differentials. ACM SIGCOMM Computer Communication Review, 40, 17-25. http://dx.doi.org/10.1145/1823844.1823847

[6] Wu, Y. and Zhou, X. (2011) Research on the IPv6 Performance Analysis Based on Dual-Protocol Stack and Tunnel Transition. 6th International Conference on ICCSE, IEEE Press, Singapore, 1091-1093. http://dx.doi.org/10.1109/ICCSE.2011.6028824

[7] Jayanthi, J.G. and Rabara, S.A. (2010) Transition and Mobility Management in the Integrated IPv4 and IPv6 Network-A Systematic Review. International Conference On ICEIE, IEEE, Kyoto, 151-162.

[8] Wu, P., Cui, Y., Wu, J.P., Liu, J. and Metz, C. (2012) Transition from IPv4 to IPv6: A State-of-the-Art Survey. IEEE Communications Surveys and Tutorials, 99, 1-18.

[9] Wu, P., Cui, Y., Xu, M., Wu, J., Li, X., Metz, C. and Wang, S. (2010) PET: Prefixing, Encapsulation and Translation for IPv4-IPv6 Coexistence. GLOBECOM 2010, IEEE Press, Miami, 1-5. http://dx.doi.org/10.1109/GLOCOM.2010.5683446

[10] Miyata, H. and Endo, M. (2010) Design and Evaluation of IPv4/IPv6 Translator for IP Based Industrial Network Protocol. 8th IEEE International Conference on INDIN, IEEE Press, Osaka, 142-147.

[11] Govil, J., Kaur, N. and Kaur, H. (2008) An Examination of IPv4 and IPv6 Networks: Constraints and Various Transition Mechanisms. IEEE Southeastcon, IEEE Press, Huntsville, 178-185. http://dx.doi.org/10.1109/SECON.2008.4494282

[12] Ge, J.G., Mi, W. and Wu, Y.L. (2014) The IPv6 Transition Mechanisms: Survey, Evaluation Criteria and Deployment Considerations. Journal of Software, 4, 896-912.

[13] Nordmark, E. (2000) Stateless IP/ICMP Translation Algorithm. IETF RFC 2765.

[14] Li, X., Bao, C.X., Chen, M., Zhang, H. and Wu, J. (2011) The CERNET IVI Translation Design and Deployment for the IPv4/IPv6 Coexistence and Transition. IETF RFC 6219.

[15] Tsirtsis, G. and Srisuresh, P. (2000) Network Address Translation-Protocol Translation (NAT-PT). IETF RFC 2766.

[16] Bagnulo, M., Matthews, P. and Van Beijnum, I. (2011) Stateful NAT64: Network Address and Protocol Translation from IPv6 Clients to IPv4 Servers. IETF RFC 6146.

[17] Huang, B., Deng, H. and Savolainen (2012) Dual-Stack Hosts Using “Bump-in-the-Host” (BIH). IETF RFC 6535.

[18] Mawatari, M., Kawashima, M. and Byrne, C. (2013) 464XLAT: Combination of Stateful and Stateless Translation. IETF RFC 6877.

[19] Bao, C., Li, X., Zhai, Y., et al. (2013) dIVI: Dual-Stateless IPv4/IPv6 Translation. IETF Draft (Work in Progress).

[20] Li, X., Bao, C., Dec, W., et al. (2013) Mapping of Address and Port Using Translation (MAP-T). IETF Draft (Work in Progress). 